\title{
Accuracy of Nonlinear Formulation of Broiler Diets: Maximizing Profits
}

http://dx.doi.org/10.1590/1516-635x1702173-180

\section{-Author(s)}

Gonçalves CA'

Almeida MA del

Faria-Júnior MJA"

Pinto MF"

Garcia-Neto M"

Post-Graduation Program of Animal Sciences of the School of Veterinary Medicine of Araçatuba, Brazil

" Professor, School of Veterinary Medicine of Araçatuba, Department of Animal Support, Production, and Health, FMVA/UNESP, Brazil

\section{Mail Address}

Corresponding author e-mail address Manoel Garcia-Neto

FMVA, Rua Clóvis Pestana 793, Bairro Dona Amélia, CEP 16050-680, Araçatuba, SP Phone: (18) 3636-1368

E-mail: mgarcia@fmva.unesp.br

\section{-Keywords}

Energy, excel, mathematical modeling, nutrition, response surface.

\section{ABSTRACT}

Nutritionists need to make commercial decisions about the optimal nutrient content broiler feeds. In order to demonstrate that broiler prices may influence dietary nutrient density, this study developed quadratic feed intake and weight gain equations, according to broiler sex and feeding phase, to be applied in a nonlinear feed formulation model. Four hundred and eighty Cobb broilers were allotted to a completely randomized experimental with six treatments, each with four replicates of 10 birds each, from 1 to 56 days old. Treatments consisted of diets containing 2800, 2900, 3000, 3100, 3200, or 3300 kcal metabolizable energy (ME)/kg and constant nutrient to $\mathrm{ME}$ ratio. A nonlinear version of the PPFR feed formulation software (http://www.fmva.unesp.br/ ppfr) was developed with the objective of optimizing energy density and bird performance. According to the results, when the models are applied in the PPFR nonlinear spreadsheet, the most favorable nutrient density content is defined by mathematical models, as optimized by the Excel Solver tool by means of cost/benefit comparisons and as a function of rearing phase (starter, grower, and finisher) and sex. This contradicts the recommendations of genetic company manuals and published requirement tables, whose goal is to maximize weight gain and do not necessarily guarantee maximum economic efficiency.

\section{INTRODUCTION}

Broiler nutritionists usually use least cost formulation programs to formulate feeds. However, this method does not optimize commercial broiler production profits (Guevara, 2004). In fact, in order to obtain the greatest economic benefits, broiler companies should apply optimization methodologies to determine the nutritional requirements, because live performance directly depend on feed intake (nutritional levels). In addition, broiler production is influenced by fluctuations in feed costs, broiler market prices, and consumer's demand for birds of different sizes (Cerrate \& Waldroup, 2009a).

Studies have shown that increasing dietary energy density promotes broiler growth and feed efficiency (Saleh et al., 2004). However, increasing energy levels result in higher production costs. In addition, considering the current rise in the price of energy sources, it is essential to review the energy levels of broiler diets.

Feed formulation using nonlinear programming takes into account practical issues. The concept of feeding by using economically optimal concentrations of nutrients is based on the law of diminishing returns (Almquist, 1953) and many different biologic and economic situations are considered in nonlinear formulation.

Aiming at addressing those challenges, mathematical models were developed in the present study to estimate broiler performance 
according to sex, age, and dietary energy density in order to develop a nonlinear version of the feeding formulation program PPFR (Garcia-Neto, 2012), which uses the Guevara (2004) model, thereby allowing to obtain the parameters of the objective function that maximizes profits.

\section{MATERIAL AND METHODS}

In this trial, 480 Cobb broilers chicks were reared between 1 and 56 days of age in a conventional broiler house, which was environmentally controlled to maintain the thermal comfort of the birds. On day 1 , birds were separated by sex, weighed, and allotted to six treatments, with four replicates of ten birds each. Replicates were equally distributed into 48 pens, each measuring $1.4 \times 3 \mathrm{~m}$, with concrete floor covered with wood-shavings litter. A continuous lighting program, with 23 hours of light daily, was applied throughout the experiment.

Diets were formulated according to the recommendations of Rostagno et al. (2005) for each sex and rearing period (starter: 1 to 21 days, grower: 22 to 42 days, and finisher: 43 to 56 days), using the feed formulation program PPFR (Garcia-Neto, 2012). The six experimental diets were based on corn-soybean meal and contained 2800, 2900, 3000, 3100, 3200, or $3300 \mathrm{kcal}$ metabolizable energy (ME)/kg. The ratio of all essential nutrients to dietary energy was maintained constant. The diets are presented in Table 1 (females) and Table 2 (males). Crumbled feed and water were offered ad libitum throughout the trial.

Live weight, feed intake, feed:energy ratio (feed intake/dietary energy content) and feed conversion ratio (feed intake/weight gain) were determined on days $21,35,42,49$, and 56 . Mortality was daily checked for the adjustment of feed intake.

Carcass quality was evaluated on days 35, 42, 49 and 56 . One bird per pen, which body weight was close to the average weight of the replicate, was selected and submitted to feed fasting for nine hours, but were offered water ad libitum. Birds were then weighed, slaughtered according to official procedures (Brasil, 1997; 1998), de-feathered, eviscerated, and chilled by immersion in cold water, after which the abdominal fat pad was manually removed and the carcasses were weighed.

Results were submitted to analysis of variance to check the effects of treatments and to obtain information on the robustness of the binomial age and nutrient density. The results were considered significant at $p<0.05$. A response surface was built to determine and quantify synergistic and antagonistic effects among treatments (Rodrigues \& lemma, 2009) using the PROC GLM of SAS statistical package (SAS Institute, 2009).

A nonlinear programming version of the PPFR software was developed with the objective of optimizing dietary energy density and bird performance. The steps of this feed formulation model that uses Excel Workbook was detailed by Guevara (2004). However, in our experiment, response surface data were used to obtain the equations according to age, sex, and dietary nutrient density in order to optimize gross profit margin in broiler feed formulation (Maximum Profit Ration), both as a nutritional strategy and as a mathematical modeling tool for broiler production. Therefore, broiler production response always requires two inputs to define the quadratic function, according to the equation:

$$
Y=f(D, E)=\beta_{0}+\beta_{1} D+\beta_{2} E+\beta_{3} D^{2}+\beta_{4} E^{2}+\beta_{5} D * E ，
$$

Where, in this study, $Y$ is the "output", i.e. the average output result (body weight, feed intake, energy consumption, feed conversion ratio, abdominal fat weight, and carcass weight); D is bird age (days); and $\mathrm{E}$ is dietary energy content (Mcal ME/kg of diet).

The linear $\left(\beta_{1} D\right.$ and $\left.\beta_{2} E\right)$ and the quadratic $\left(\beta_{3} D^{2}\right.$ and $\left.\beta_{4} E^{2}\right)$ responses and the possible effects of the interactions $\left(\beta_{5} D^{*} E\right)$ follow the law of diminishing returns for age and energy content ("Inputs").

According to the procedure of Guevara (2004), the model identifies which is the best fit of parameters to maximize profits and to promote the best animal performance (source of income). However, it does not necessarily maximize their potential weight gain because it takes into account product price (price per kilogram of live broiler paid by the market) and cost (feed expenses), and therefore, the diets will contain the nutrient and energy levels that are the most appropriate in each specific scenario.

\section{RESULTS AND DISCUSSION}

Dietary energy content is the most important item in broiler feed formulation, and makes up $70 \%$ of the total diet (Guevara, 2004; Saleh et al., 2004). Therefore, this study aimed at understanding and determining the optimal energy content required to accelerate broiler growth, without, however, compromising maximum economic return. 
Table 1 - Ingredients and nutritional composition of female diets, according to rearing phase.

\begin{tabular}{|c|c|c|c|c|c|c|c|c|c|c|c|c|c|c|c|c|c|c|}
\hline Ingredients (\%) & & Star & $\operatorname{ter}(1-2$ & $1 \mathrm{~d}$ of & age) & & & Grow & $\operatorname{ler}(22-$ & $42 \mathrm{~d}$ of & f age) & & & Finish & er (43- & $-56 d$ of & f age) & \\
\hline Inert & 3.17 & 0.00 & 0.00 & 0.00 & 0.00 & 0.00 & 6.53 & 3.22 & 0.00 & 0.00 & 0.00 & 0.00 & 8.76 & 5.52 & 2.27 & 0.00 & 0.00 & 0.00 \\
\hline Corn & 60.93 & 62.54 & 57.57 & 52.59 & 47.61 & 42.64 & 66.92 & 69.31 & 71.48 & 66.89 & 62.30 & 57.71 & 70.53 & 73.05 & 75.57 & 76.03 & 71.73 & 67.43 \\
\hline Soybean oil & 0.00 & 0.19 & 2.61 & 5.04 & 7.46 & 9.89 & 0.00 & 0.00 & 0.07 & 2.43 & 4.78 & 7.14 & 0.00 & 0.00 & 0.00 & 0.69 & 2.99 & 5.29 \\
\hline Soybean meal - $45 \%$ & 32.01 & 33.25 & 35.69 & 38.13 & 40.57 & 43.01 & 23.22 & 24.05 & 24.92 & 27.07 & 29.22 & 31.36 & 18.15 & 18.80 & 19.44 & 20.48 & 22.41 & 24.34 \\
\hline Dicalcium phosphate & 1.61 & 1.67 & 1.74 & 1.82 & 1.89 & 1.97 & 1.32 & 1.37 & 1.42 & 1.48 & 1.54 & 1.61 & 1.07 & 1.11 & 1.15 & 1.19 & 1.25 & 1.31 \\
\hline Common salt & 0.44 & 0.46 & 0.47 & 0.49 & 0.51 & 0.53 & 0.39 & 0.40 & 0.41 & 0.43 & 0.45 & 0.46 & 0.34 & 0.35 & 0.36 & 0.38 & 0.39 & 0.41 \\
\hline L-Lysine $\mathrm{HCl}$ & 0.22 & 0.23 & 0.21 & 0.19 & 0.18 & 0.16 & 0.20 & 0.20 & 0.21 & 0.19 & 0.17 & 0.15 & 0.10 & 0.11 & 0.11 & 0.11 & 0.09 & 0.06 \\
\hline DL- Methionine & 0.24 & 0.24 & 0.26 & 0.28 & 0.30 & 0.32 & 0.16 & 0.16 & 0.17 & 0.18 & 0.20 & 0.21 & 0.06 & 0.06 & 0.07 & 0.07 & 0.08 & 0.09 \\
\hline L- Threonine & 0.05 & 0.06 & 0.06 & 0.06 & 0.06 & 0.06 & 0.03 & 0.03 & 0.03 & 0.03 & 0.03 & 0.03 & 0.00 & 0.00 & 0.00 & 0.00 & 0.00 & 0.00 \\
\hline Calcitic limestone & 0.82 & 0.85 & 0.86 & 0.88 & 0.89 & 0.91 & 0.73 & 0.75 & 0.78 & 0.79 & 0.80 & 0.82 & 0.65 & 0.67 & 0.70 & 0.72 & 0.73 & 0.73 \\
\hline Vitamin and Mineral premix ${ }^{A}$ & 0.52 & 0.52 & 0.52 & 0.52 & 0.52 & 0.52 & 0.51 & 0.51 & 0.51 & 0.51 & 0.51 & 0.51 & 0.33 & 0.33 & 0.33 & 0.33 & 0.33 & 0.33 \\
\hline
\end{tabular}

Calculated composition

Metabolizable Energy (kcal/kg) $28002900 \quad 3000310032003300 \quad 280029003000310032003300 \quad 280029003000310032003300$ Crude Protein (\%) $\quad 19.9220 .6321 .3222 .0122 .7023 .3916 .3516 .9317 .5218 .1018 .6819 .2714 .1814 .6915 .2015 .7016 .2116 .72$ \begin{tabular}{llllllllllllllllllll}
\hline Calcium (\%) & 0.80 & 0.83 & 0.86 & 0.89 & 0.92 & 0.95 & 0.68 & 0.70 & 0.73 & 0.75 & 0.78 & 0.80 & 0.58 & 0.60 & 0.62 & 0.64 & 0.66 & 0.68
\end{tabular} Available phosphorus (\%) $\quad \begin{array}{lllllllllllllllllll}0.40 & 0.42 & 0.43 & 0.45 & 0.46 & 0.48 & 0.34 & 0.35 & 0.36 & 0.38 & 0.39 & 0.40 & 0.29 & 0.30 & 0.31 & 0.32 & 0.33 & 0.34\end{array}$ \begin{tabular}{lllllllllllllllllllll}
\hline Potassium (\%) & 0.76 & 0.78 & 0.81 & 0.85 & 0.88 & 0.91 & 0.61 & 0.63 & 0.66 & 0.68 & 0.71 & 0.74 & 0.53 & 0.55 & 0.57 & 0.59 & 0.61 & 0.63
\end{tabular} Sodium (\%) $\quad \begin{array}{llllllllllllllllll}0.19 & 0.20 & 0.21 & 0.21 & 0.22 & 0.23 & 0.17 & 0.18 & 0.18 & 0.19 & 0.20 & 0.20 & 0.15 & 0.16 & 0.16 & 0.17 & 0.17 & 0.18\end{array}$ \begin{tabular}{lllllllllllllllllllllll}
\hline Chlorine (\%) & 0.35 & 0.36 & 0.37 & 0.38 & 0.38 & 0.39 & 0.31 & 0.32 & 0.34 & 0.34 & 0.35 & 0.35 & 0.27 & 0.28 & 0.29 & 0.29 & 0.30 & 0.30
\end{tabular}

\begin{tabular}{lllllllllllllllllllll}
\hline Linoleic Acid (\%) & 1.33 & 1.47 & 2.70 & 3.94 & 5.17 & 6.40 & 1.38 & 1.43 & 1.52 & 2.71 & 3.91 & 5.11 & 1.41 & 1.46 & 1.51 & 1.90 & 3.08 & 4.25
\end{tabular}

Dig. Lysine (\%) $\quad \begin{array}{lllllllllllllllllll} & 1.11 & 1.15 & 1.19 & 1.23 & 1.27 & 1.31 & 0.88 & 0.91 & 0.94 & 0.97 & 1.00 & 1.04 & 0.69 & 0.71 & 0.74 & 0.76 & 0.79 & 0.81\end{array}$

\begin{tabular}{llllllllllllllllllll}
\hline Dig. Methionine (\%) & 0.51 & 0.53 & 0.56 & 0.58 & 0.60 & 0.63 & 0.40 & 0.41 & 0.42 & 0.44 & 0.46 & 0.48 & 0.28 & 0.29 & 0.30 & 0.31 & 0.33 & 0.34
\end{tabular}

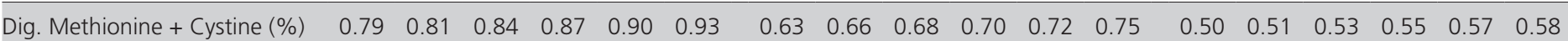

\begin{tabular}{lllllllllllllllllllll}
\hline Dig. Tryptophan (\%) & 0.22 & 0.22 & 0.23 & 0.25 & 0.26 & 0.27 & 0.17 & 0.18 & 0.18 & 0.19 & 0.20 & 0.21 & 0.14 & 0.15 & 0.15 & 0.16 & 0.17 & 0.18
\end{tabular}

Dig. Threonine (\%) $\quad \begin{array}{llllllllllllllllllll}0.72 & 0.75 & 0.77 & 0.80 & 0.82 & 0.85 & 0.57 & 0.59 & 0.61 & 0.63 & 0.65 & 0.67 & 0.48 & 0.49 & 0.51 & 0.53 & 0.55 & 0.56\end{array}$

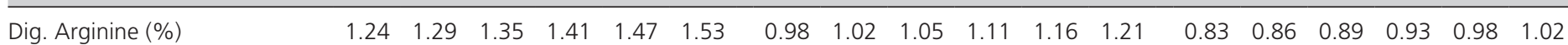

Dig. Valine (\%) $\quad \begin{array}{llllllllllllllllll}0.83 & 0.86 & 0.89 & 0.92 & 0.95 & 0.98 & 0.68 & 0.71 & 0.73 & 0.76 & 0.78 & 0.81 & 0.60 & 0.62 & 0.64 & 0.66 & 0.68 & 0.71\end{array}$

Dig. Isoleucine (\%) $\begin{array}{llllllllllllllllll}0.77 & 0.80 & 0.83 & 0.87 & 0.90 & 0.94 & 0.62 & 0.64 & 0.66 & 0.69 & 0.72 & 0.75 & 0.53 & 0.55 & 0.57 & 0.59 & 0.62 & 0.64\end{array}$

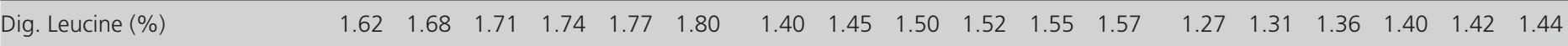

\begin{tabular}{|c|}
\hline Dig. His \\
\hline
\end{tabular}

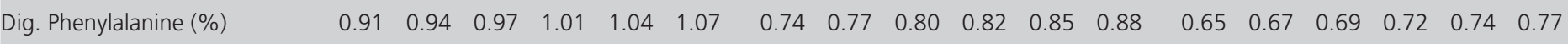

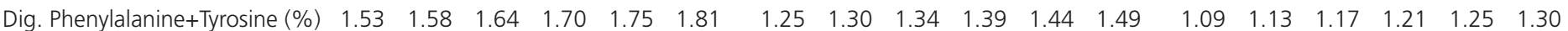
Energy:Protein Ratio $\quad 140.5140 .6140 .7140 .8140 .9141 .1 \quad 171.3171 .3171 .3171 .3171 .3171 .3 \quad 197.4197 .4197 .4197 .4197 .4197 .4$

A Vitamin and Mineral supplement supplied according to rearing phase (amount/kg of product) included: Starter: vit, A - 1,670,000 U.I; vit, D3 - 335,000 U.I; vit, E - 2,500 mg; vit, K3 - 417 mg; vit, B1 - 250 mg; vit, B2 - 835 mg; vit, B6 - 250 mg; vit, B12 - 2,000 mcg; folic acid - 100 mg; biotin - 9 mg; nicotinic acid - 5,835 mg; Pantothenic acid - 1,870 mg; Cu - 1,000 mg; Co - 17 mg; I 170 mg; Fe - 8,335 mg; Mn - 10,835mg; Zn - 7,500 mg; Se - 35 mg; Choline chloride 50\% - 116,670 mg; Methionine - 250,000 mg; Coccidiostat- 13,335 mg; Growth Promoter- 13,335 mg; Antioxidant - 2,000 mg, Grower: vit, A - 1,335,000 U.l; vit, D3 - 300,000 U.l; vit, E - 2,000 mg; vit, K3 - 335 mg; vit, B1 - 167 mg; vit, B2 - 670 mg; vit, B6 - 170 mg; vit, B12 - 1,670 mcg; folic acid - 67 mg; biotin - 7 mg; nicotinic acid - 4,670 mg; Pantothenic acid - 1,870 mg; Cu - 1,000 mg; Co - 17 mg; I 170 mg; Fe - 8,335 mg; Mn - 10,835 mg; Zn - 7,500 mg; Se - 35 mg; Choline chloride 50\% - 83,340mg; Methionine - 235,000mg; Coccidiostat - 10,000 mg; Growth Promoter- 10,000mg; Antioxidant - 2,000mg, Finisher: vit, A - 1,670,000 U.I; vit, D3 - 335,000 U.l; vit, E - 2,335 mg; vit, K3 - 400 mg; vit, B1 - 100 mg; vit, B2 - 800 mg; vit, B6 - 200 mg; vit, B12 - 2,000 mcg; folic acid - 67 mg; biotin - 7 mg; nicotinic acid - 5,670 mg; Pantothenic acid- 2,000 mg; Cu - 2,000 mg; Co - 27 mg; I - 270 mg; Fe - 16,670 mg; Mn - 17,335 mg; Zn - 12,000 mg; Se - 70 mg; Choline chloride 50\% - 100,000mg; Methionine - 235,000mg; Antioxidant - 2,000 mg. 
Table 2 - Ingredients and nutritional composition of male diets, according to rearing phase.

\begin{tabular}{|c|c|c|c|c|c|c|c|c|c|c|c|c|c|c|c|c|c|c|}
\hline \multirow{2}{*}{$\begin{array}{l}\text { Ingredients (\%) } \\
\text { Inert } \\
\end{array}$} & \multicolumn{6}{|c|}{ Starter (1-21 d of age) } & \multicolumn{6}{|c|}{ Grower (22-42 d of age) } & \multicolumn{6}{|c|}{ Finisher (43-56 d of age) } \\
\hline & 2.22 & 0.00 & 0.00 & 0.00 & 0.00 & 0.00 & 5.30 & 1.94 & 0.00 & 0.00 & 0.00 & 0.00 & 8.12 & 4.85 & 1.59 & 0.00 & 0.00 & 0.00 \\
\hline Corn & 60.79 & 61.89 & 56.89 & 51.89 & 46.89 & 41.89 & 64.65 & 66.96 & 66.30 & 61.62 & 56.93 & 52.25 & 69.24 & 71.71 & 74.19 & 73.16 & 68.83 & 64.51 \\
\hline oybean oil & 0.00 & 0.37 & 2.80 & 5.23 & 7.66 & 10.09 & 0.00 & 0.00 & 1.01 & 3.38 & 5.75 & 8.12 & 0.00 & 0.00 & 0.00 & 1.19 & 3.49 & 5.80 \\
\hline oybean meal - 45\% & 32.20 & 33.55 & 36.00 & 38.45 & 40.90 & 43.35 & 26.56 & 27.512 & 29.00 & 31.21 & 33.43 & 35.65 & 19.74 & 20.44 & 21.15 & 22.49 & 24.43 & 26.36 \\
\hline Dicalcium phosphate & 1.71 & 1.78 & 1.86 & 1.94 & 2.02 & 2.10 & 1.39 & 1.44 & 1.50 & 1.57 & 1.63 & 1.70 & 1.15 & 1.19 & 1.23 & 1.28 & 1.34 & 1.40 \\
\hline Common salt & 0.47 & 0.48 & 0.50 & 0.52 & 0.54 & 0.56 & 0.41 & 0.42 & 0.44 & 0.45 & 0.47 & 0.49 & 0.36 & 0.37 & 0.38 & 0.40 & 0.41 & 0.43 \\
\hline -Threonine & 0.05 & 0.06 & 0.06 & 0.06 & 0.06 & 0.06 & 0.03 & 0.03 & 0.03 & 0.03 & 0.03 & 0.04 & 0.03 & 0.03 & 0.03 & 0.03 & 0.03 & 0.03 \\
\hline Limestone & 0.87 & 0.89 & 0.91 & 0.93 & 0.94 & 0.96 & 0.76 & 0.79 & 0.81 & 0.82 & 0.83 & 0.85 & 0.68 & 0.70 & 0.72 & 0.74 & 0.75 & 0.76 \\
\hline Vitamin and Mineral premix ${ }^{A}$ & 0.52 & 0.52 & 0.52 & 0.52 & 0.52 & 0.52 & 0.51 & 0.51 & 0.51 & 0.51 & 0.51 & 0.51 & 0.33 & 0.33 & 0.33 & 0.33 & 0.33 & 0.33 \\
\hline \multicolumn{19}{|l|}{ Calculated composition } \\
\hline Metabolizable Energy (kc & 2800 & 2900 & 3000 & 3100 & 3200 & 3300 & 2800 & 2900 & 3000 & 3100 & 3200 & 3300 & 2800 & 2900 & 3000 & 3100 & 3200 & 3300 \\
\hline otassium (\%) & 0.76 & 0.79 & 0.82 & 0.85 & 0.88 & 0.91 & 0.67 & 0.69 & 0.72 & 0.74 & 0.77 & 0.80 & 0.56 & 0.57 & 0.59 & 0.62 & 0.64 & 0.66 \\
\hline Sodium (\%) & 0.20 & 0.21 & 0.22 & 0.23 & 0.23 & 0.24 & 0.18 & 0.19 & 0.19 & 0.20 & 0.21 & 0.21 & 0.16 & 0.17 & 0.17 & 0.18 & 0.18 & 0.19 \\
\hline Chlorine (\%) & 0.37 & 0.38 & 0.39 & 0.39 & 0.40 & 0.41 & 0.33 & 0.34 & 0.35 & 0.35 & 0.36 & 0.36 & 0.30 & 0.31 & 0.32 & 0.33 & 0.33 & 0.34 \\
\hline noleic Acid (\%) & 1.33 & 1.55 & 2.79 & 4.03 & 26 & 6.50 & 1.36 & 1.41 & 1.95 & 3.16 & 4.37 & 57 & 1.40 & 1.45 & 1.50 & 2.13 & 3.31 & 4.48 \\
\hline ig. Lysine (\%) & 1.11 & 1.15 & 1.19 & 1.23 & 1.27 & 1.31 & 0.96 & 0.99 & 1.03 & 1.06 & 1.10 & 1.13 & 0.81 & 0.84 & 0.87 & 0.90 & 0.93 & 0.95 \\
\hline ig. Methionine (\%) & 0.52 & 0.53 & 0.56 & 0.58 & 0.61 & 0.63 & 0.44 & 0.46 & 0.47 & 0.49 & 0.52 & 0.54 & 0.36 & 0.37 & 0.39 & 0.40 & 0.42 & 0.44 \\
\hline Dig. Methionine + Cystine (\%) & 0.79 & 0.82 & 0.85 & 0.87 & 0.90 & 0.93 & 0.69 & 0.72 & 0.74 & 0.76 & 0.79 & 0.81 & 0.58 & 0.60 & 0.62 & 0.65 & 0.67 & 0.69 \\
\hline Dig. Tryptophan (\%) & 0.22 & 0.22 & 0.24 & 0.25 & 0.26 & 0.27 & 0.19 & 0.19 & 0.20 & 0.21 & 0.22 & 0.23 & 0.15 & 0.16 & 0.16 & 0.17 & 0.18 & 0.19 \\
\hline Dig. Threonine (\%) & 0.72 & 0.75 & 0.77 & 0.80 & 0.83 & 0.85 & 0.62 & 0.65 & 0.67 & 0.69 & 0.71 & 0.74 & 0.53 & 0.54 & 0.56 & 0.58 & 0.60 & 0.62 \\
\hline ig. Arginine (\%) & 1.25 & 1.30 & 1.36 & 1.42 & 1.48 & 1.54 & 1.08 & 1.12 & 1.17 & 1.22 & 1.27 & 1.33 & 0.88 & 0.91 & 0.94 & 0.98 & 1.03 & 1.08 \\
\hline
\end{tabular}

A Vitamin and Mineral supplement used in the rations in three rearing phases (amount/kg of product) included: Starter: vit, A - 1,670,000 U.I; vit, D3 - 335,000 U.I; vit, E - 2,500 mg; vit, K3 - 417 mg; vit, B1 - 250 mg; vit, B2 - 835 mg; vit, B6 - 250 mg; vit, B12 - 2,000 mcg; folic acid - 100 mg; biotin - 9 mg; nicotinic acid - 5,835 mg; Pantothenic acid - 1,870 mg; Cu - 1,000 mg; Co - 17 mg; I - 170 mg; Fe - 8,335 mg; Mn - 10,835mg; Zn - 7,500 mg; Se - 35 mg; Choline chloride 50\% - 116,670 mg; Methionine - 250,000 mg; Coccidiostat- 13,335 mg; Growth Promoter - 13,335 mg; Antioxidant - 2,000 mg, Growth: vit, A - 1,335,000 U.l; vit, D3 - 300,000 U.l; vit, E - 2,000 mg; vit, K3 - 335 mg; vit, B1 - 167 mg; vit, B2 - 670 mg; vit, B6 - 170 mg; vit, B12 - 1,670 mcg; folic acid - 67 mg; biotin - 7 mg; nicotinic acid - 4,670 mg; Pantothenic acid - 1,870 mg; Cu - 1,000 mg; Co - 17 mg; I - 170 mg; Fe - 8,335 mg; Mn - 10,835 mg; Zn - 7,500 mg; Se - 35 mg; Choline chloride 50\% - 83,340mg; Methionine - 235,000mg; Coccidiostat - 10,000 mg; Growth Promoter- 10,000mg; Antioxidant - 2,000mg, Finisher: vit, A - 1,670,000 U.I; vit, D3 - 335,000 U.l; vit, E - 2,335 mg; vit, K3 - 400 mg; vit, B1 - 100 mg; vit, B2 - 800 mg; vit, B6 - 200 mg; vit, B12 - 2,000 mcg; folic acid - 67 mg; biotin - 7 mg; nicotinic acid - 5,670 mg; Pantothenic acid- 2,000 mg; Cu - 2,000 mg; Co - 27 mg; I - 270 mg; Fe - 16,670 mg; Mn - 17,335 mg; Zn - 12,000 mg; Se - 70 mg; Choline chloride 50\% 100,000mg; Methionine - 235,000mg; Antioxidant - 2,000 mg.

Live weight and feed intake results of male and female broilers are presented separately for the periods of 1 to 20 days of age and 21 to 56 days of age. This strategy was adopted to allow a better fit to the growth curve of broilers, which is typically sigmoidal.

There were significant interactions between age and dietary metabolizable energy content for live weight and feed intake of both males and females (Table 3). Therefore, in order to determine the best market age to obtain the greatest profit, diet nutrient density must be considered, and it depends on bird age.

The effects of age and nutrient density (independent variables) on the dependent variables (e.g. body weight) are complex and cannot be explained by a simple linear function. The models developed in the present study were shown to be biologically realistic and capable of explaining the results, as demonstrated by the obtained coefficients of determination $\left(R^{2}\right)$, 
Table 3 - Probability, coefficient of variation (CV), coefficient of determination $\left(R^{2}\right)$ and analysis of variance of live weight (LW) and feed intake (FI) of female and male broilers between 1 to 20 days of age and 21 to 56 days of age.

\begin{tabular}{|c|c|c|c|c|c|c|c|c|}
\hline \multirow{3}{*}{$\begin{array}{l}\text { Parameter } \\
\operatorname{Pr}>|t| \text { value }\end{array}$} & \multicolumn{4}{|c|}{1 to $20 \mathrm{~d}$ of age } & \multicolumn{4}{|c|}{21 to $56 \mathrm{~d}$ of age } \\
\hline & \multicolumn{2}{|c|}{ LW } & \multicolumn{2}{|c|}{$\mathrm{FI}$} & \multicolumn{2}{|c|}{ LW } & \multicolumn{2}{|c|}{$\mathrm{Fl}$} \\
\hline & Females & Males & Females & Males & Females & Males & Females & Males \\
\hline Intercept & 0.0948 & 0.0979 & 0.2333 & 0.2036 & $<0.0001$ & $<0.0001$ & $<0.0001$ & $<0.0001$ \\
\hline Metabolizable energy (ME) & 0.0845 & 0.0879 & 0.2357 & 0.2059 & $<0.0001$ & $<0.0001$ & $<0.0001$ & $<0.0001$ \\
\hline Age & 0.1263 & 0.0476 & 0.0444 & 0.0897 & $<0.0001$ & 0.0067 & $<0.0001$ & $<0.0001$ \\
\hline$M E * M E$ & 0.9793 & 0.0822 & 0.2470 & 0.2159 & $<0.0001$ & $<0.0001$ & $<0.0001$ & $<0.0001$ \\
\hline Age*ME & 0.0052 & 0.0039 & 0.0712 & 0.0756 & 0.4862 & 0.0341 & 0.0033 & $<0.0001$ \\
\hline Age ${ }^{*}$ Age & $<0.0001$ & $<0.0001$ & $<0.0001$ & $<0.0001$ & 0.0095 & 0.0497 & 0.0001 & $<0.0001$ \\
\hline CV (\%) & 7.7559 & 9.3033 & 9.4586 & 10.6080 & 2.6408 & 2.8283 & 2.8130 & 1.6739 \\
\hline$R^{2}$ & 0.9943 & 0.9921 & 0.9947 & 0.9938 & 0.9968 & 0.9966 & 0.9973 & 0.9990 \\
\hline \multicolumn{9}{|l|}{ Analysis of variance } \\
\hline \multicolumn{9}{|l|}{ Causes of variation $(\mathrm{Pr}>\mathrm{F})$} \\
\hline Linear & $<0.0001$ & $<0.0001$ & $<0.0001$ & $<0.0001$ & $<0.0001$ & $<0.0001$ & $<0.0001$ & $<0.0001$ \\
\hline Quadratic & $<0.0001$ & $<0.0001$ & $<0.0001$ & $<0.0001$ & $<0.0001$ & 0.0001 & $<0.0001$ & $<0.0001$ \\
\hline Total Model & $<0.0001$ & $<0.0001$ & $<0.0001$ & $<0.0001$ & $<0.0001$ & $<0.0001$ & $<0.0001$ & $<0.0001$ \\
\hline
\end{tabular}

which were extremely high (>0.95). Moreover, the coefficients of variation (CV) indicate that the instability of responses was very low, except for abdominal fat weight (Table 4), which is known to be very unstable (Sampaio, 2007).

The analysis of variance (Table 3 and 4) of the evaluated parameters as function of age and dietary metabolizable energy content show that the mathematical models were highly significant ( $p$ $<0.0001)$ for all studied parameters, indicating that the experimental data fully fit the models.
Therefore, the analysis of the response surface allows defining an optimal region of dietary nutrient density according to broiler age and sex instead of a best-value point, as in the case of linear formulation. As a practical application, it allows reducing dietary nutrient density without compromising broiler performance when weight gain and feed intake are considered, and this is precisely what promotes the economic viability of the nonlinear principle.

The females, which have lower genetic potential for muscle accretion than males, presented higher

Table 4 - Probability, coefficient of variation (CV), coefficient of determination $\left(\mathrm{R}^{2}\right)$ and analysis of variance for energy consumption, feed conversion, abdominal fat weight and carcass weight responses of female and male broilers.

\begin{tabular}{|c|c|c|c|c|c|c|c|c|}
\hline \multirow{3}{*}{$\begin{array}{l}\text { Parameter } \\
\text { Pr> }|t| \text { value }\end{array}$} & \multicolumn{4}{|c|}{21 to $56 \mathrm{~d}$ of age } & \multicolumn{4}{|c|}{35 to $56 \mathrm{~d}$ of age } \\
\hline & \multicolumn{2}{|c|}{ Energy consumption } & \multicolumn{2}{|c|}{ Feed conversion } & \multicolumn{2}{|c|}{ Abdominal fat weight } & \multicolumn{2}{|c|}{ Carcass weight } \\
\hline & Females & Males & Females & Males & Females & Males & Females & Males \\
\hline Intercept & $<0.0001$ & $<0.0001$ & 0.0014 & $<0.0001$ & 0.8831 & 0.3019 & 0.0095 & 0.1232 \\
\hline Metabolizable energy (ME) & $<0.0001$ & $<0.0001$ & 0.0278 & 0.0008 & 0.7151 & 0.2235 & 0.0100 & 0.0965 \\
\hline Age & 0.0647 & 0.1624 & 0.3561 & 0.4390 & 0.1189 & 0.4565 & 0.5121 & 0.4938 \\
\hline$M E * M E$ & $<0.0001$ & $<0.0001$ & 0.1053 & 0.0057 & 0.5787 & 0.1673 & 0.0126 & 0.1078 \\
\hline Age ${ }^{\star M E}$ & 0.0244 & 0.0177 & 0.2410 & 0.0500 & 0.0951 & 0.3034 & 0.7017 & 0.9290 \\
\hline Age ${ }^{*}$ Age & $<0.0001$ & $<0.0001$ & 0.7995 & 0.2009 & 0.1721 & 0.4511 & 0.1621 & 0.0066 \\
\hline CV $(\%)$ & 4.5415 & 5.0530 & 1.4666 & 1.5728 & 28.7591 & 36.8360 & 8.9750 & 10.7504 \\
\hline $\mathrm{R}^{2}$ & 0.9915 & 0.9898 & 0.9896 & 0.9860 & 0.6908 & 0.5235 & 0.9036 & 0.8873 \\
\hline \multicolumn{9}{|l|}{ Analysis of variance } \\
\hline \multicolumn{9}{|l|}{ Causes of variation $(\mathrm{Pr}>\mathrm{F})$} \\
\hline Linear & $<0.0001$ & $<0.0001$ & $<0.0001$ & $<0.0001$ & $<0.0001$ & $<0.0001$ & $<0.0001$ & $<0.0001$ \\
\hline Quadratic & $<0.0001$ & $<0.0001$ & 0.2544 & 0.0110 & 0.3364 & 0.2899 & 0.0174 & 0.0074 \\
\hline Total Model & $<0.0001$ & $<0.0001$ & $<0.0001$ & $<0.0001$ & $<0.0001$ & $<0.0001$ & $<0.0001$ & $<0.0001$ \\
\hline
\end{tabular}


abdominal fat content (Figure 3 ) and it almost doubled between the two evaluated periods (1-42 and 1-56 days old). The excessive abdominal fat deposition shows that there was a waste of energy. Therefore, the efficiency of energy utilization of females is poor after 40 days, as shown by the significantly lower carcass yield and greater deposition of abdominal fat compared with males. Consequently, females should be slaughtered at an earlier age for economic reasons. In addition, the results show that the influence of dietary nutrient density on live weight is reduced as broilers age (Figure 2), as demonstrated by the steeper slope of the body weight curve when broilers are younger. This indicates that the optimal slaughter weight depends on bird sex (higher profits for males), justifying the earlier slaughter of females. Also, the interaction between age and nutrient density shows that targeting only weight gain in feed formulation (least cost formulation) is costly, contradicting the law of diminishing returns. Therefore, in order to minimize costs and time and to maximize economic returns, it is essential to apply the nonlinear principle as a tool for formulation.

The simulations obtained with calibration of the current model, using specific PPFR spreadsheets for males and females, were more consistent when compared with previous studies that considered only a single diet for both sexes (Miller et al., 1986; Guevara 2004). When a single diet is applied, the performance of females fed on diets formulated for males is not compromised, but there are economic losses. On the other hand, the performance of males fed on female diets is reduced, also leading to economic losses. In addition, considering carcass quality, the ratio between energy and nutrients must be maintained constant so that body composition does not change as body weight increases, thereby maintaining carcass quality (Saleh et al., 2004; Cerrate \& Waldroup, 2009a; 2009b). That is the reason why the model "energy to nutrient" ratio is considered the best method for nonlinear feed formulation (Cerrate \& Waldroup, 2009a; 2009b), and also because metabolizable energy is the main factor that affects feed intake, inducing an increase or reduction of the intake of the other nutrients (Leeson et al., 1996).

It is also observed (Table 4) that the energy consumption (ME* $\mathrm{ME}$ ) response was curvilinear. Therefore, the model must be nonlinear to ensure the maximization of economic efficiency (Guevara, 2004; Cerrate \& Waldroup, 2009a; Eila et al., 2012). Consequently, the most favorable nutrient density content, when nonlinear PPFR formulation is applied, will be defined by mathematical models optimized by the Excel Solver tool, using cost/benefit comparisons according to rearing phase (starter, grower, and finisher) and sex. This contradicts the recommendations of the genetic line manuals and published requirement tables, whose goal is to maximize weight gain and do not necessarily ensure maximum economic efficiency.

The results of the present study obtained by applying the models to the nonlinear PPFR spreadsheet answer the questions mentioned above and proved to be reliable within the range of values based on which the equations were generated ( $1-56$ days old). Moreover, when feedstuff and chicken prices change, the model seeks to maintain the most favorable nutrient density to achieve maximum profit by relocating the other ingredients available (Guevara, 2004; Renz, 2005).

This economic fit is very dependent on how narrow or broad is the energy to nutrient ratio, i.e., because prestarter and starter diets typically have very close ratios (e.g., energy to protein ratio of 140:1; Tables 1 and 2), they do not allow extensive optimization manipulations by the Solver. However, because feed intake is still low during those phases (Figure 1), such limitation does not cause significant damage. In addition, as birds age and energy to protein ratios in the formulation increase (to 171:1 in the grower phase and 197:1 in the finisher phase; Tables 1 and 2, respectively), nonlinear programming of more flexible ratios, allows adjustements precisely as feed intake becomes increasingly relevant, having direct impact on costs.

The obtained results clearly show the importance of developing mathematical models and applying new feed formulation principles that take into account the current knowledge on nutrient utilization and tissue deposition in modern broilers, particularly of protein and energy, aiming at optimizing nutrient carcass deposition (Lopez et al., 2007). Therefore, mathematical modeling presents an opportunity for the broiler industry to produce better-quality carcasses for increasingly demanding consumers, who seek lowfat food products (Meinerz et al., 2001), and at the same time, to balance the cost-benefit ratio.

\section{CONCLUSIONS}

The results of this study suggest that, when applying the concept of precision feeding for broilers, nonlinear feed formulation provides better results compared with linear feed formulation because the requirements of all nutrients are automatically adjusted by the mathematical model, resulting in optimal dietary energy supply, in addition to estimating the most profitable weight gain. 
Gonçalves CA, Almeida MA de,

Faria-Júnior JA, Pinto MF, Garcia-Neto M
Accuracy of Nonlinear Formulation of Broiler Diets: Maximizing Profits

b

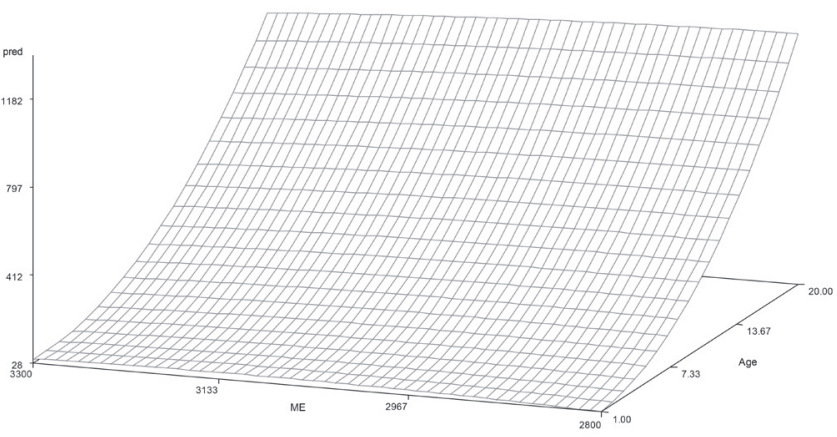

b

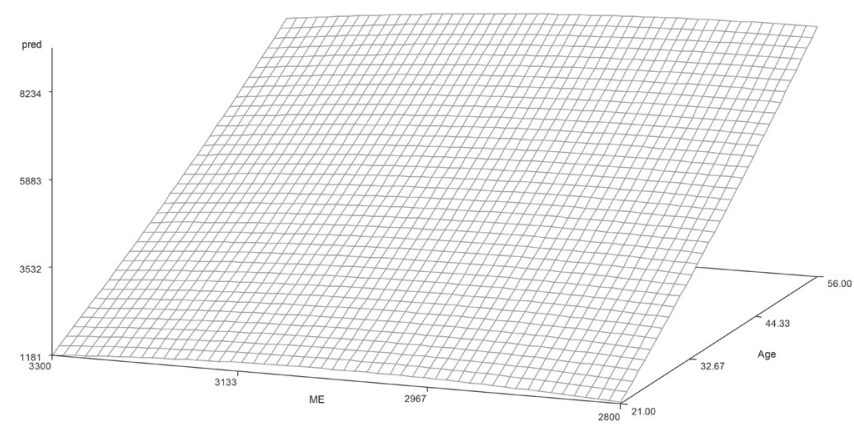

Figure 1 - Response Surface of feed intake ( $\mathrm{g}$ ) of females (a) and males (b), according to age (days) and dietary energy density (kcal ME/kg).

a

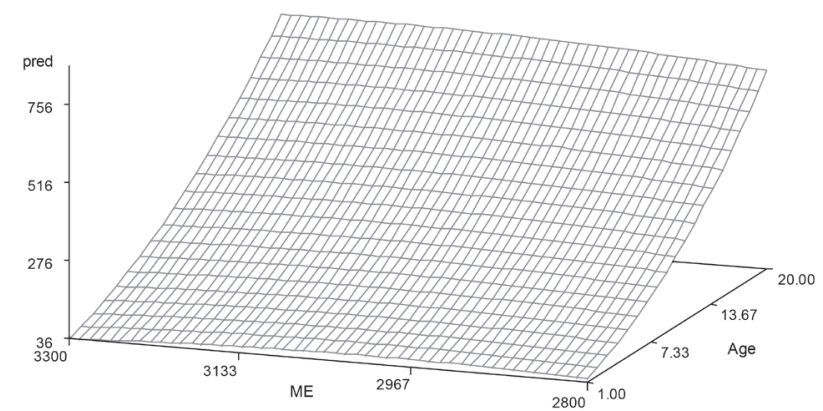

a

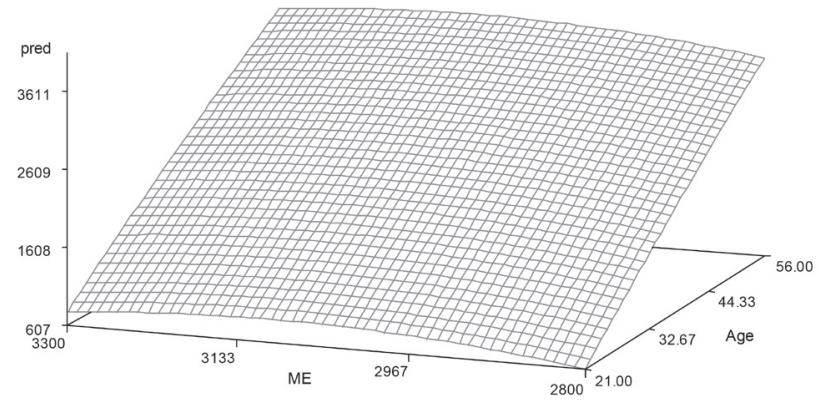

b

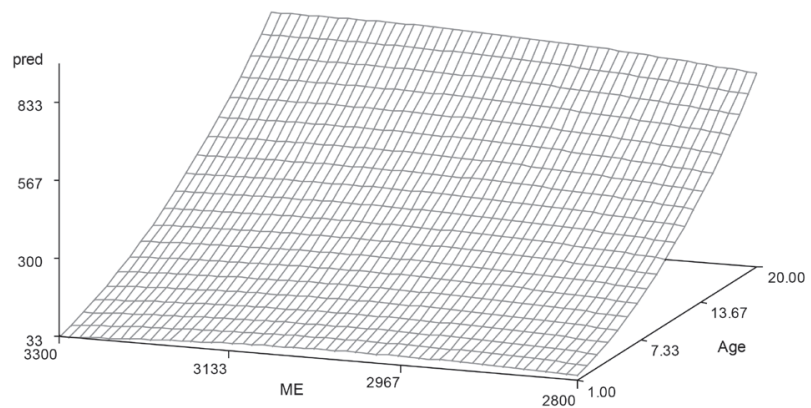

b

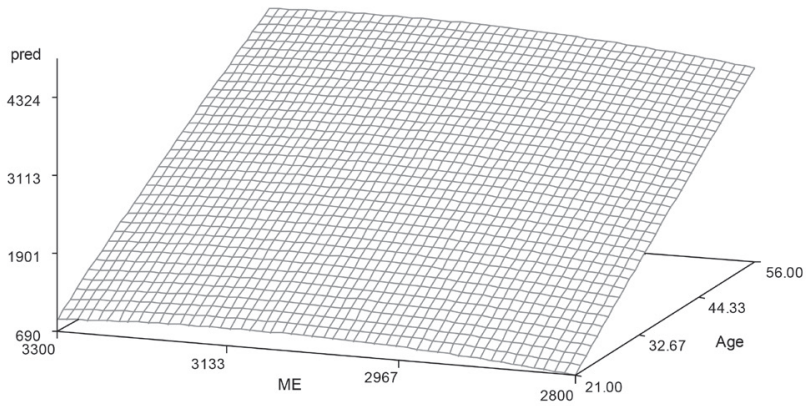

Figure 2 - Response Surface of body weight (g) of females (a) and males (b), according to age (days) and dietary energy density (kcal ME/kg). 
a

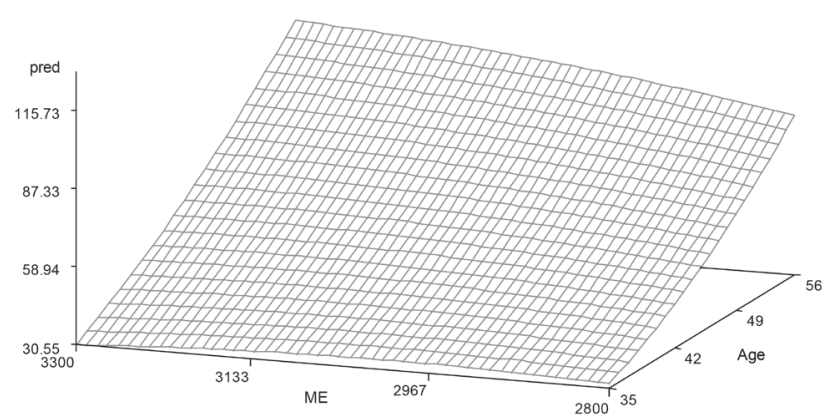

b

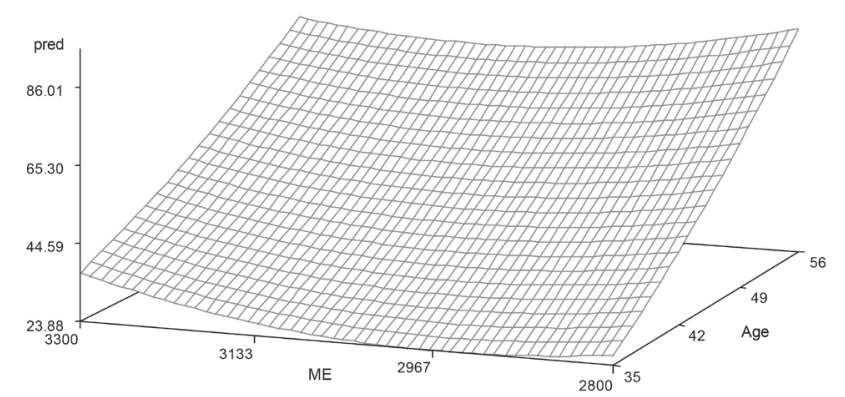

Figure 3 - Response Surface of abdominal fat weight ( $\mathrm{g}$ ) of females (a) and males (b), according to age (days) and dietary energy density (kcal ME/kg).

\section{ACKNOWLEDGEMENTS}

To the São Paulo Research Foundation (FAPESP) by financial support and CAPES Foundation for the master scholarship granted to the first author.

\section{REFERENCES}

Almquist HJ. Interpretation of amino acid requirement data according to the law of diminishing returns. Archives of Biochemistry and Biophysics $1953 ; 44: 245-247$.

Brasil. Ministério da Agricultura, Pecuária e Abastecimento. Portaria $n^{\circ}$ 210, de 10 de novembro de 1998. Aprova o regulamento técnico da inspeção tecnológica e higiênico-sanitária de carne de aves. Diário oficial [da] República Federativa do Brasil, poder Executivo, Brasília, DF, 26 nov. 1998. Seção 1, p. 226.

Brasil. Decreto $n^{\circ}$. 2244, de 5 de junho de 1997. Estabelece regulamentação da inspeção industrial e sanitária de produtos de origem animal. Diário oficial [da] República Federativa do Brasil, poder Executivo, Brasília, DF, 4 jun.1997. Seção 11, p. 204.

Cerrate S, Waldroup P. Maximum profit feed formulation of broilers: 1 . Development of a feeding program model to predict profitability using non linear programming. International Journal of Poultry Science 2009a;8:205-215.

Cerrate S, Waldroup P. Maximum Profit Feed Formulation of Broilers: 2. Comparison among Different Nutritional Models. International Journal of Poultry Science 2009b;8:216-228.

Eila N, Lavvaf A, Farahvash T. A model for obtaining more economic diets for laying hen. African Journal of Agricultural Research 2012;7(8):13021306.

Garcia-Neto M. PPFR: programa prático para formulação e otimização de ração frangos de corte programação não linear [cited 2012 Fev 15].Available from:: https://sites.google.com/site/ ppfrprogramforfeedformulation/.

Guevara VR. Use of Nonlinear Programming to Optimize Performance Response to Energy Density in Broiler Feed Formulation. Poultry Science 2004;83:147-151.
Kessler AM, Snizek Júnior PN, Brugalli I. Manipulação da quantidade de gordura na carcaça de frango. Anais da Conferência Apinco 2000 de Ciência e Tecnologia Avícolas; 2000; Santos, São Paulo. Brasil. Campinas: FACTA; 2000. v. 1, p. 107-134.

Leeson S, Caston L, Summers JD. Broiler response to energy diet. Poultry Science 1996;75:529-535.

Lopez G, Lange K, Leeson S. Partitioning of retained energy in broilers and birds with intermediate growth rate. Poultry Science 2007;86:21622171.

Meinerz C, Ribeiro AML, Penz-Junior AM, Kessler AM. Níveis de energia e peletização no desempenho e rendimento de carcaça de frangos de corte com oferta alimentar equalizada. Revista Brasileira de Zootecnia 2001;30:2026-2032.

Miller BR, Arraes RA, Pesti GM. Formulation of broiler finishing ratios by quadratic programming. Southern Journal of Agricultural Economics 1986;18(1):141-150.

Renz SV. Comparação de sistemas de formulação lineares e não lineares para frangos de corte [dissertação]. Porto Alegre (RS): Universidade Federal do Rio Grande do Sul; 2005.

Rodrigues MS, lemma AF. Planejamento de experimentos e otimização de processos. $2^{a}$ ed. Campinas: Casa do Espírito Amigo Fraternidade Fé e Amor; 2009. 358p.

Rostagno HS, Albino LFT, Donzele JL, Gomes PC, Oliveira RF, Lopes DC, et al. Tabelas brasileiras para aves e suínos: composição de alimentos e exigências nutricionais. $2^{\mathrm{a} e d}$. Viçosa: UFV; 2005. 186p.

Saleh EA, Watkins SE, Waldroup AL, Waldroup PW. Effects of dietary nutrient density on performance and carcass quality of male broilers grown for further processing. International Journal of Poultry Science 2004;3:1-10.

Sampaio IBM. Estatística aplicada à experimentação animal. 3ed. Belo Horizonte: Fundação de Estudo e Pesquisa em Medicina Veterinária e Zootecnia; 2007. 264 p

SAS Institute. SAS user's guide: statistics. version five edition. Cary, NC; 2009. 
In the article entitled Litter Accuracy of Nonlinear Formulation of Broiler Diets: Maximizing Profits published in the Revista Brasileira de Ciência Avícolas/ Brazilian Journal of Poultry Science, v17 (2):173-180, in page 173 where it was written

\section{EAuthor(s)}

Gonçalves CA'

Almeida MA del

Faria-Júnior JA"

Pinto MF"

Garcia-Neto $M^{\|}$

the correct form is

\section{-Author(s)}

Gonçalves $C A^{\prime}$

Almeida MA de'

Faria-Júnior MJA"

Pinto MF"

Garcia-Neto M"

http://dx.doi.org/10.1590/1516-635x1702173-180 